\title{
Quadriceps femoris cross-sectional area and specific leg strength: relationship between different muscles and squat variations
}

\author{
Filip Kojić ${ }^{1}$, Saša Đurić ${ }^{\text {Corresp., } 2 \text {, Igor Ranisavljev }}{ }^{3}$, Stanimir Stojiljković ${ }^{3}$, Vladimir Ilić ${ }^{3}$ \\ ${ }^{1}$ Teachers Education Faculty, University of Belgrade, Belgrade, Serbia \\ 2 Faculty of Sport, University of Ljubljana, Ljubljana, Slovenia \\ 3 Faculty of Sport and Physical Education, University of Belgrade, Belgrade, Serbia \\ Corresponding Author: Saša Đurić \\ Email address: sasa.djuric@fsp.uni-lj.si
}

Background. The aim was to determine the relationship between the cross-sectional area of the quadriceps femoris and strength performance in the deep and parallel barbell squat.

Methods. The sample included 16 university students ( 7 female, $24.1 \pm 1.7$ years). Muscle strength was expressed as external load, including the one-repetition maximum and the body mass segments involved (calculated according to Dempster's method). The cross-sectional area of the quadriceps femoris muscles was determined using ultrasound, while leg muscle mass was measured using the Bioelectrical Impedance method.

Results. The cross-sectional areas of the three vastii muscles and leg muscle mass showed moderate to strong correlation with external load in both squat types $(r=0.509-0.873)$. However, partial correlation ( cross-sectional area of quadriceps femoris muscles were controlled) showed significant association only between leg muscle mass and deep squat $(r=0.64, p<0.05)$. The cross-sectional area of the vastus lateralis showed a slightly higher correlation with external load in the parallel than in the deep squat $(r=0.67, p<0.01$ vs. $r=0.59, p<0.05)$. The regression analysis extracted the vastus medialis crosssectional area as the most important factor in manifesting strength (parallel squat: $R^{2}=0.569$; deep squat: $R^{2}=0.499$, both $p<0.01$ ). The obtained results suggest that parallel squat strength depends mainly on the cross-sectional area of the vastii muscles, while it seems that the performance in the deep squat requires an additional engagement of the hip and back extensor muscle groups. 
1 Quadriceps femoris cross-sectional area and specific 2 leg strength: relationship between different muscles 3 and squat variations 


\section{Abstract}

5 Background. The aim was to determine the relationship between the cross-sectional area of the 6 quadriceps femoris and strength performance in the deep and parallel barbell squat.

7 Methods. The sample included 16 university students ( 7 female, $24.1 \pm 1.7$ years). Muscle

8 strength was expressed as external load, including the one-repetition maximum and the body

9 mass segments involved (calculated according to Dempster's method). The cross-sectional area

10 of the quadriceps femoris muscles was determined using ultrasound, while leg muscle mass was 11 measured using the Bioelectrical Impedance method.

12 Results. The cross-sectional areas of the three vastii muscles and leg muscle mass showed moderate to strong correlation with external load in both squat types $(\mathrm{r}=0.509-0.873)$. However, partial correlation (cross-sectional area of quadriceps femoris muscles were controlled) showed significant association only between leg muscle mass and deep squat $(\mathrm{r}=0.64, \mathrm{p}<0.05)$. The cross-sectional area of the vastus lateralis showed a slightly higher correlation with external load in the parallel than in the deep squat ( $r=0.67, \mathrm{p}<0.01$ vs. $\mathrm{r}=0.59, \mathrm{p}<0.05)$. The regression analysis extracted the vastus medialis cross-sectional area as the most important factor in manifesting strength (parallel squat: $\mathrm{R}^{2}=0.569$; deep squat: $\mathrm{R}^{2}=0.499$, both $\mathrm{p}<0.01$ ). The obtained results suggest that parallel squat strength depends mainly on the cross-sectional area of the vastii muscles, while it seems that the performance in the deep squat requires an additional engagement of the hip and back extensor muscle groups. 


\section{Introduction}

24 The squat is one of the most popular and important exercises for developing strength and power and is often integrated into strength and conditioning training (Toutoungi, Lu, Leardini, Catani, \& O'Connor, 2000; Escamilla, 2001). The exercise is performed in a closed kinetic chain involving the hip, knee, and ankle joints and requires a significant level of hip and ankle mobility as well as stability of the lumbar spine (Kritz, Cronin, \& Hume, 2009). It is generally believed that the quadriceps femoris (QF) muscles, assuming 4 pennate muscles (rectus femoris-RF, vastus intermedius-VI, vastus medialis-VM and vastus lateralis-VL), make the greatest contribution to the squat motion, while the hip extensors, hip abductors, and ankle plantar flexors also play important roles (Schoenfeld, 2010).

In resistance training, there are numerous variations of the squat, such as the foot position, the position of the dumbbell (load), or the range of motion (Schoenfeld, 2010; Clark, Lambert, \& Hunter, 2012). The range of motion (ROM) during the squat, known as the knee flexion angle, is frequently modified in practice, with three types of squats most commonly used: 1) partial squat with ROM of 0 - 45 degrees $\left({ }^{\circ}\right), 2$ ) parallel squat with knee flexion around $90^{\circ}$, and 3) deep squat with ROM over $100^{\circ}$ (Clark et al., 2012). It has been shown that different ROM squat variations produce different dynamic and kinematic changes (Drinkwater, Moore, \& Bird, 2012), possibly indicating that engagement of upper-leg muscles could be dependent on movement amplitude prescription. The studies on the variability of muscle activation during different ROM squat variations mostly utilized electromyographic (EMG) analyses, and these reports are mostly inconclusive. While some findings indicate that a change in the amplitude of flexion does not cause changes in QF muscle activity (Contreras, Vigotsky, Schoenfeld, Beardsley, \& Cronin, 2016; da Silva et al., 2017), others indicate a change in EMG activity of different QF muscle groups as a function of squat ROM (Caterisano et al., 2002; Marchetti et al., 2016). For example, Contreras et al. (2016) reported similar EMG responses of VL and the hip extensor muscles (gluteus maximus and biceps femoris) during both parallel and deep squats in resistance-trained females. However, in resistance-trained males, shorter variants of the squat (partial and parallel) elicited greater involvement of the VL, VM and RF muscles, while conversely the EMG amplitude of the gluteus maximus was greater during deep squats (Caterisano et al., 2002; Marchetti et al., 2016). The shortcoming of the aforementioned studies is that the contribution of the VI muscle is neglected because estimation of the EMG signals of the deep muscles, such as 
54 VI, cannot be performed directly with surface electrodes. The exception is the distal portion of 55 the VI muscle, which is the only area available for surface EMG but can only be used for 56 isometric contraction at low force levels (Watanabe \& Akima, 2011). In addition, the majority of

57 EMG studies have measured total muscle electrical activity and have not determined the partial 58 contribution of the muscular and neural components. Considering that different squat modalities 59 could accentuate intramuscular hypertrophy of the quadriceps (Earp, Newton, Cormie, \&

60

61

62 63

64 65 66 67 68 69 70 71 72 73 74 75 76 77 78 79 80

\section{1}

82

83 84 Blazevich, 2015; Kubo, Ikebukuro, \& Yata, 2019), which in turn could lead to different performance adaptations (Mangine et al., 2014; Wilhelm et al., 2014; Methenitis et al., 2016) and alter potential knee injury risks (Zebis, Andersen, Bencke, Kjaer, \& Aagaard, 2009; Toumi et al., 2013; Mangine et al., 2014a), it is extremely important to evaluate and confirm the relationship between the development of different quadriceps muscle parts and squat strength in two fundamental ROM variations (i.e. parallel vs. deep squat).

Although there are a considerable number of studies in the current literature that investigated the relationship between lower-body muscle size and squat strength (Häkkinen et al., 1998; Secomb et al., 2015; Seitz et al., 2016), most of them measured the outer thigh volume and did not provide data on the contribution of each quadriceps muscle parts in different ROMs squat performance. It should also be noted that previous research has assessed squat strength by onerepetition maximum test (1RM), which only reflects the weight lifted and does not take into account the mass of the other segments of the body (i.e., head, trunk, and upper-leg). Hence, this could be a confounding factor when determining total QF muscle resistance, and incorporating both lifted weight and body segments would be more a reliable indicator of external load.

Therefore, the aim of the study was to evaluate the relationship between the cross-sectional area of four quadriceps femoris muscles and external load measured during two major squat variations: deep squat and parallel squat. We hypothesized that different parts of the quadriceps muscle will have a different relationship with maximum strength in squats and that contribution of vastii muscles (VM, VL and VI) would be greater during parallel compared to deep squat.

\section{Materials \& Methods} Sample and study design

The sample included 16 university volunteers (9 males and 7 females) with no experience of resistance training. During the sample selection, the study was advertised to all departments at 
85 the university (Table 1). Both sexes were included to reduce gender sampling disparity in sports

86 science and medical research (Costello, Bieuzen, \& Bleakley, 2014).

87

88

89

90

91

92

93

94

95

96

97

98

99

100

101

102

103

104

105

106

107

108

109

110

111

112

113

114

115
INSERT TABLE 1 HERE

Participants were not professional athletes and did not suffer from leg or back injuries. All participants voluntarily participated in the study and previously signed a written informed consent regarding the experimental procedure and potential risks. The study was approved by the Ethics Committee of the Faculty of Sport and Physical Education, University of Belgrade (protocol number: 2316/19-2) and conducted in accordance with the Declaration of Helsinki.

The strength tests were performed on two separate days, with the deep barbell squat on the first day and the parallel barbell squat on the second day. Before strength testing, two familiarization sessions were performed, where subjects received instructions on the proper squat technique for the deep and parallel squat exercises. During familiarization sessions, the subjects performed 2 controlled sets with 8 to 10 reps of both squat variants with barbell on the shoulders, while being continuously monitored and corrected by experienced coach. Both squat variations were performed with the feet at shoulder width and with toes pointed forward or slightly outward. The barbell was placed in the high bar position across the shoulders on the trapezius, slightly above the acromion. To eliminate the negative impact of fatigue, the tests took place 48 hours apart. Both tests were performed at approximately the same time of day (12-14h). Between each test and retest session, the subjects had $48 \mathrm{~h}$ of rest, in order to avoid the effects of fatigue. Anthropometry, body composition and quadriceps femoris (QF) cross-sectional measures were taken two days prior to first strength testing. Subjects were advised to avoid any form of physical activity 48 hours prior to the testing, as well as not to consume alcoholic or caffeinated beverages 12 hours before testing. The study protocol is shown in Figure 1.

\section{INSERT FIGURE 1 HERE}

\section{Variables and protocols}

Strength in both squat variations was assessed by one-repetition maximum test (1RM) using the standardized testing protocol (Baechle \& Earle, 2008). The test was preceded by a 10-minute warm-up (light running and warm up exercises), followed by 8-10 repetitions of the exercise

PeerJ reviewing PDF | (2021:06:62995:3:0:NEW 12 Oct 2021) 
116 with a load of $50 \% 1 \mathrm{RM}$ and 2-3 repetitions of the exercise with a load of $60-80 \% 1 \mathrm{RM}$. Each 117 subject had 5 attempts to lift the maximum weight. Rest intervals between trials were set at 3 118 minutes. Deep squat was performed with maximum amplitude of flexion in the knee joint. The 119 required knee angle in this position was 45 degrees (Contreras et al., 2016; Kubo et al., 2019), 120 with the lumbar spine kept neutral. The height of the deep squat was measured for each 121 participant individually. An elastic band was then stretched, which the participants had to touch 122 during the squat to achieve the required angle of 45 degrees at the knee. Parallel squat was 123 performed in a position where the femurs were parallel to the ground when the trochanter mayor 124 and lateral epicondyle of the femur were at the same level (Figure 2; Earp et al., 2015; Contreras 125 et al., 2016; da Silva et al., 2017). External load was calculated as the sum of 1RM and mass of 126 body segments (head, trunk, arms and thighs) using the model proposed by Dempster \& 127 Gaughran (1967). To determine the repeatability of the strength measurement, test-retest was 128 conducted on ten participants on the two separate days.

129

130

131

132 133

134

135

136

137

138

139

140

141

142

143

144

145

146

\section{INSERT FIGURE 2 HERE}

Body height was measured with a Martin's portable anthropometer (Siber-Hegner, Switzerland) to an accuracy of $0.1 \mathrm{~cm}$. Body composition variables, including skeletal muscle mass (SMM), percent body fat (PBF), and dominant leg muscle mass (LSMM), were measured with In-Body 720 (Biospace Co., Seoul, Korea) using Direct Segmental Multi frequency-Bioelectrical Impedance Analysis (DSM-BIA method). Prior to testing, the subjects were instructed not to eat anything in the morning, avoid any kind of exercise 24 before body composition analyze and perform all physiological needs before the measurement. Subjects were in the standing position for at least 5 minutes prior to measurement for redistribution of body fluids. During the measurement all subjects were in light sport clothing and had no metal accessories. The subjects individually picked their dominant leg, based on the question "Which leg would you use to shoot the ball?" (van Melick et al., 2017) and that variable was used for further analysis.

Image acquisition was performed by the experienced and previously trained radiology specialist. The cross-sectional area (CSA) was measured using an ultrasound scanner (Siemens Antares, Erlangen, Germany), with a linear probe of $7.5 \mathrm{MHz}$ and expressed in square millimetres $\left(\mathrm{mm}^{2}\right)$. The $2 \mathrm{D}$ ellipse diagnostic method was applied. Imaging was conducted after about 10 min rest in 
147 supine position on right leg in full extension. The ultrasound probe was transversally placed on 148 the skin with minimal compression with conductive gel used for acoustic coupling on the skins 149 surface. The CSA of the rectus femoris (RF) was measured at the level of the three-fifths of the 150 distance from the anterior superior iliac spine to the superior patellar border (Seymour et al., 151 2009), while the vastus intermedius (VI) CSA measurement was performed at one-half between 152 spina iliaca anterior superior and the proximal border of the patella (Haaf et al., 2017). The VL

153

154

155

156

157

158

159

160

161

162

163

164

165

166

167

168

169

170

171

172

173

174

175

176

177

muscle CSA was measured at $50 \%$ of femur length, defined as the distance of $50 \%$ between the greater trochanter and the lateral condyle (Franchi et al., 2018). The vastus medialis (VM) was measured at the level of the distal portion above the medial side of patella. To determine the repeatability of the ultrasound measurement, test-retest was conducted on ten participants on the two separate days.

\section{Statistics}

The Shapiro-Wilk and Levene'ş tests were used to assess the normality of the distribution and the homogeneity of variances, respectively. Reliability of the strength and ultrasound measures were accessed using the intra-class correlation coefficient (ICC). The independent samples t-test was used to determine the differences between male and female CSA of muscles and LSMM. To analyse the effects of sex and differences between external load of deep squat and parallel squat, the 2-way ANOVA (model: Mixed between-within subjects) was used. Pearson's moment correlation was used to examine the association between external load, LSMM and CSA of 4 muscles forming QF (RF, VI, VM and VL). A partial correlation (controlling for QF muscles size) was performed to determine the influence of other lower-body muscle groups, except QF, on external load during both squat variations. The relationships were classified as trivial $(<0.4)$, moderate $(0.4-0.6)$, strong $(0.6-0.8)$, very strong $(0.8-1.0)$, and perfect correlation (1.0) (Dancey \& Reidy, 2007). For discussion purposes, the correlation coefficients were directly compared with their 95\% confidence intervals (Vigotsky et al., 2019). Additionally, to find the best predictive model of the quadriceps muscles for the external load of deep and parallel squat, a backward multiple regression was applied. Statistical analysis was performed using the IBM SPSS Statistics software package (Version 21, SPSS Inc, Chicago, IL, USA). All data are presented as means $\pm S D$, where $p \leq 0.05$ was considered a statistically significant determinant. 


\section{Results}

179 Excellent reliability was observed for both ultrasound (RF: ICC $=0.997, \mathrm{CI}=0.990-0.999, \mathrm{p}<$

180 0.001; VI: $\mathrm{ICC}=0.995, \mathrm{CI}=0.979-0.998, \mathrm{p}<0.001 ; \mathrm{VM}=0.997, \mathrm{CI}=0.987-0.999, \mathrm{p}<0.001$;

181 VL: $\mathrm{ICC}=0.996, \mathrm{CI}=0.982-0.999, \mathrm{p}<0.001)$ and strength (parallel squat: $\mathrm{ICC}=0.997, \mathrm{CI}=$

182 0.987-0.999, $\mathrm{p}<0.001$; deep squat: $\mathrm{ICC}=0.997, \mathrm{CI}=0.990-0.999, \mathrm{p}<0.001)$ measurements.

183 As expected, the CSA of QF muscle and LSMM was higher in males than females $(\mathrm{t}=3.449$, $\mathrm{df}$

$184=14, \mathrm{p}=0.004, \mathrm{ES}=1.75$ and $\mathrm{t}=5.824, \mathrm{df}=14, \mathrm{p}=0.000, \mathrm{ES}=2.89$, respectively). Males

185 demonstrated significantly higher CSA of $\mathrm{RF}(\mathrm{t}=2.238, \mathrm{df}=14, \mathrm{p}=0.042, \mathrm{ES}=1.11), \mathrm{VI}(\mathrm{t}=$

186 3.626, $\mathrm{df}=14, \mathrm{p}=0.003, \mathrm{ES}=1.89)$ and $\mathrm{VM}(\mathrm{t}=3.283, \mathrm{df}=14, \mathrm{p}=0.005, \mathrm{ES}=1.71)$. A

187 significant difference was not found only for $\mathrm{VL}(\mathrm{t}=1.749, \mathrm{df}=14, \mathrm{p}=0.102, \mathrm{ES}=0.92)$,

188 although in females the average CSA of VL was lower for about $55 \mathrm{~mm}^{2}$ (Table 2).

189

190

\section{INSERT TABLE 2 HERE}

191

192

Male subjects achieved significantly higher external load for deep and parallel squat $(\mathrm{F}=24.882$, 193 $\mathrm{df}=1, \mathrm{p}=0.000, \mathrm{ES}=1.33$ ). Subjects of both sexes demonstrated significantly higher external

194

195

196

197

198

199

200

201

202

203

204

205

206

207 load for parallel compared to deep squat $(F=44.136, \mathrm{df}=1, \mathrm{p}=0.000, \mathrm{ES}=1.77)$. As the interaction between sex and squat variation was not statistically significant $(\mathrm{F}=0.036, \mathrm{df}=1, \mathrm{p}$ $=0.852, \mathrm{ES}=0.05)$, this proves that strength manifests in the same manner in both genders (Figure 3). 
208 Even when a CSA of QF was controlled, LSMM was significantly correlated with deep $(\mathrm{r}=$ $2090.641, \mathrm{p}=0.026)$, but not with parallel squat $(\mathrm{r}=0.530, \mathrm{p}=0.076)$.

210

211

\section{INSERT TABLE 3 HERE}

212

213 Further regression analysis revealed that of the four predictors of quadriceps muscles, the best-fit 214 model highlighted VM as the most important factor in the manifestation of strength in both squat 215 variations. For external load of parallel squat, VM explained about $57 \%$ of variance $\left(\mathrm{R}^{2}=0.569\right.$, $216 \mathrm{p}=0.001$ ), with the equation for this model: $\mathrm{y}=0.3349 \mathrm{x}+61.365$. On the other hand, the VM 217 model explained about $50 \%$ of the external load deep squat $\left(\mathrm{R}^{2}=0.499, \mathrm{p}=0.002\right)$, with the 218 equation: $\mathrm{y}=0.3114 \mathrm{x}+58.609$ (Figure 4).

219

220

\section{INSERT FIGURE 4 HERE}

221

222

\section{Discussion}

223

224

225

226

227

228

229

230

231

232

233

234

235

236

237

238

The study was conducted with the aim to determine contribution of quadriceps muscle parts in manifestation of strength during deep and parallel barbell squat. The present results indicate that there is a significant correlation between the CSA of QF and 1RM strength in both squat variations, but a stronger association was found with PS. Squat performance is largely dependent on the CSA of all three vastii muscles, with VM dimensions playing a significant role in the manifestation of strength in both squat variations. The contribution of VL CSA appears to be more pronounced in the parallel compared to the deep squat.

We observed a significant association between lower-body muscle size and maximal strength in both squat variations. In addition, males demonstrated larger CSA of QF muscles and LSMM, as well as higher values of external load for both squat types. This implies that larger muscle sizes in males are the fundamental reason for significantly higher strength for deep and parallel squat. Pearson correlation coefficient revealed moderate to strong association between muscle size and squat strength $(\mathrm{r}=0.58-0.68)$. Similar results were obtained by Suchomel \& Stone (2017) $(r=0.64)$ and Häkkinen et al. (1998) $(\mathrm{r}=0.56-0.64)$ for males and females, indicating that QF muscle mass plays an important role in squat strength performance, however additional factors are also important (i.e. other muscle groups, neural activity, muscle architecture). These statements are

PeerJ reviewing PDF | (2021:06:62995:3:0:NEW 12 Oct 2021) 
239 further confirmed by our results, as squat external load is more related to total leg muscle mass 240 compared to the QF CSA. Moreover, QF CSA showed a rather stronger relationship with parallel 241 than with deep squat ( $r=0.68, p<0.01$ vs. $r=0.59, p<0.05$, respectively), while conversely, when 242 CSA of QF was controlled, LSMM was significantly correlated only with deep squat.

243 Considering that ROM significantly determines strength output in the squat (Drinkwater et al., 244 2012), we can assume that in addition to the QF, the other leg muscle groups are also 245 significantly involved in the performance of squats with larger ROMs. Previous training studies 246 with deep and half squats, showed that performing deep squats $\left(\mathrm{ROM} 0^{\circ}-140^{\circ}\right)$ influence higher 247 relative increases in squat $1 \mathrm{RM}$ and significantly affect the volume of adductor and gluteus 248 maximus muscles, in comparison to half squats (Kubo et al., 2019). However, the level of 249 quadriceps hypertrophy was similar in both groups. On the other hand, Bloomquist et al. (2013) 250 251 252 253 254 255 256 257 258 demonstrated greater QF hypertrophy after full ROM squats $\left(0^{\circ}-120^{\circ}\right)$ compared to partial $\left(0^{\circ}\right.$ $60^{\circ}$ ). These partial disagreements could be explained by different squats ROM prescription, which might affect the level of stimulus on different leg muscle groups. In contrast to the vastii muscles, RF showed no significant association with 1RM in either squat variation. Apart from knee extension, RF acts as a hip flexor (Robertson, Wilson, \& St Pierre, 2008), and it is quite possible that single-joint knee extension exercises are more suitable for training the RF (Ema, Sakaguchi, Akagi, \& Kawakami, 2016; Kubo et al., 2019). The CSA of the VM showed the highest correlation with both deep and parallel squat, indicating a crucial role of VM muscle size in squat performance. From a practical point of view, this is of great importance considering that knee stability depends primarily on VM function and that VM asymmetry, as well as VM atrophy, lead to various knee problems including patelo-femoral pain syndrome (Toumi et al., 2013). The relationship between the CSA of VL and parallel squat was stronger compared to deep squat ( $\mathrm{r}=0.65, \mathrm{p}<0.01$ vs. $\mathrm{r}=0.55, \mathrm{p}<0.05$ ), explaining why the CSA of total QF was more strongly correlated with the external load of parallel squat. In addition, both Brechue \& Abe (2002) and Secomb et al. (2015) reported that the thickness of VL was a strong predictor of $1 \mathrm{RM}$ in back squat and squat jump performance, while Earp et al. (2010) indicated that leg power production and jumping performance were strongly dependent on the architecture of the gastrocnemius lateralis muscle rather than VL. Nimphius, McGuigan, \& Newton (2012) showed a moderate relationship between VL muscle thickness and relative squat $1 \mathrm{RM}$ and emphasized that the performance of the muscle is strongly dependent on its 
270 architecture, including muscle fascicle length and pennation angle. The complexity of the leg 271 strength and power performance phenomenon in multi-joint movements consisting of hip, knee, 272 and ankle is even greater when the total EMG activity during squat variations is analysed. Using 273 the muscle EMG approach in squat analysis, Contreras et al. (2016) and Caterisano et al. (2002) 274 reported that the EMG values of VL and VM showed no significant differences during DS, PS 275 and front squat. Moreover, EMG measurements support the importance of the hip extensors, 276 especially the gluteus maximus in DS strength output, whose EMG amplitude showed a 277 significant increase with squat depth (Caterisano et al., 2002).

278

\section{Strengths and limitations of the study}

280 The main strength of this study is that this is the first study to evaluate the contribution of each 281 quadriceps muscle part in different ROMs of squat performance. Most previous studies only

282

283

284

285

286

287

288

289

290

291

292

293

294

295

296

297

298

299

300 measured outer thigh volume and did not provide this information. Moreover, we were able to evaluate the contribution of the VI, which was neglected in previous studies. Another strength of this study is that it provides more accurate information about muscle strength by calculating the exact external load, taking into account the mass of the other body segments in addition to the 1RM. This is very important when calculating muscle strength as it could be a confounding factor in determining total QF muscle strength.

A limitation of the present study investigating CSA and strength in deep and parallel squat includes the lack of data on the neural contraction component and muscle architecture. In addition, the CSA of all three vasti muscles (VI, VM and VL) was measured in the distal region. Previous studies have shown that CSA varies depending on the measurement position (Noorkoiv, Nosaka, \& Blazevich, 2010), so there is a possibility of partial variation in results, and we recommend that future studies include measurement of QF in the proximal, middle, and distal regions. The second limitation refers to the strength testing protocol, as the order of the tests was fixed, which could have resulted in increased fatigue from the first test session influencing strength in the second session. Therefore we strongly encourage that future studies apply a randomised strength testing design. Also, it should be noted that this is cross sectional research, based on correlation analysis, and longitudinal training studies are required to better explore how specific hypertrophy of QF regions is affected by squat depth variations. In addition, subjects require optimal hip and ankle mobility to perform deep squat safely and successfully, so this type 
301 of exercise is not suitable for everyone in practice (Contreras et al., 2016). Therefore,

302 standardization of depth during deep squat is difficult to establish among the subjects, mainly

303 due to varying levels of joint mobility and muscle flexibility. We instructed our subjects to go as

304 deep as possible and reach $45^{\circ}$ in the knees while keeping the lumbar spine neutral, which was

305 quite difficult task for some subjects.

306

307 Conclusions

308 In conclusion, the results prove that the external load in the parallel and deep squat is highly

309 dependent on the CSA of the quadriceps femoris, while a slightly stronger association was found

310 in the parallel squat. The CSA of the VM has a fundamental role in manifesting strength in both

311 types of squat and the highest correlation with both squat variations. VL showed a slightly higher

312 correlation with parallel squat external load. The vastus intermedius showed an intermediate

313 correlation with both variants of the squat, but slightly lower than VM and VL. We can assume

314 that muscle strength during the parallel squat depends mainly on the CSA of three vastii muscles,

315 while the deep squat performance requires additional function of other muscle groups, especially

316 the hip and back extensors. From a practical point of view, the inclusion of both squat variations

317 in resistance training program might be the best possible approach, while supplementary

318 exercises should be implemented for optimal training of the rectus femoris due to the multi-joint

319 function of this muscle.

320

321

322 References

323 Baechle TR, Earle RW. 2008. Essentials of strength training and conditioning. Human kinetics

324 Bloomquist K, Langberg H, Karlsen S, Madsgaard S, Boesen M, Raastad T. 2013. Effect of

325 range of motion in heavy load squatting on muscle and tendon adaptations. European Journal of

326 Applied Physiology 113 (8):2133-2142. doi:10.1007/s00421-013-2642-7

327 Brechue WF, Abe T. 2002. The role of FFM accumulation and skeletal muscle architecture in

328 powerlifting performance. European Journal of Applied Physiology 86 (4):327-336

329 Caterisano A, Moss RF, Pellinger TK, Woodruff K, Lewis VC, Booth W, Khadra T. 2002. The

330 effect of back squat depth on the EMG activity of 4 superficial hip and thigh muscles. Journal of

331 Strength and Conditioning Research 16 (3):428-432

332 Clark DR, Lambert MI, Hunter AM. 2012. Muscle activation in the loaded free barbell squat: a

333 brief review. Journal of Strength and Conditioning Research 26 (4):1169-1178.

334 doi:10.1519/JSC.0b013e31822d533d 
335 Contreras B, Vigotsky AD, Schoenfeld BJ, Beardsley C, Cronin J. 2016. A Comparison of 336 Gluteus Maximus, Biceps Femoris, and Vastus Lateralis Electromyography Amplitude in the 337 Parallel, Full, and Front Squat Variations in Resistance-Trained Females. Journal of Applied 338 Biomechanics 32 (1):16-22. doi:10.1123/jab.2015-0113

339 Costello JT, Bieuzen F, Bleakley CM. 2014. Where are all the female participants in Sports and 340 Exercise Medicine research? European Journal of Sport Science 14 (8):847-851.

341 doi:10.1080/17461391.2014.911354

342 da Silva JJ, Schoenfeld BJ, Marchetti PN, Pecoraro SL, Greve JMD, Marchetti PH. 2017.

343 Muscle Activation Differs Between Partial and Full Back Squat Exercise With External Load

344 Equated. Journal of Strength and Conditioning Research 31 (6):1688-1693.

345 doi:10.1519/jsc.0000000000001713

346 Dancey CP, Reidy J. 2007. Statistics without maths for psychology. Pearson education,

347 Dempster WT, Gaughran GR. 1967. Properties of body segments based on size and weight.

348 American Journal of Anatomy 120 (1):33-54

349 Drinkwater EJ, Moore NR, Bird SP. 2012. Effects of changing from full range of motion to

350 partial range of motion on squat kinetics. Journal of Strength and Conditioning Research 26

351 (4):890-896. doi:10.1519/JSC.0b013e318248ad2e

352 e Lima KM, da Matta TT, de Oliveira LF. 2012. Reliability of the rectus femoris muscle cross-

353 sectional area measurements by ultrasonography. Clinical Physiology and Functional Imaging

35432 (3):221-226. doi:10.1111/j.1475-097X.2011.01115.x

355 Earp JE, Joseph M, Kraemer WJ, Newton RU, Comstock BA, Fragala MS, Dunn-Lewis C,

356 Solomon-Hill G, Penwell ZR, Powell MD, Volek JS, Denegar CR, Häkkinen K, Maresh CM.

357 2010. Lower-body muscle structure and its role in jump performance during squat,

358 countermovement, and depth drop jumps. Journal of Strength and Conditioning Research 24

359 (3):722-729. doi:10.1519/JSC.0b013e3181d32c04

360 Earp JE, Newton RU, Cormie P, Blazevich AJ. 2015. Inhomogeneous Quadriceps Femoris

361 Hypertrophy in Response to Strength and Power Training. Medicine and Science in Sports and

362 Exercise 47 (11):2389-2397. doi:10.1249/mss.0000000000000669

363 Ema R, Sakaguchi M, Akagi R, Kawakami Y. 2016. Unique activation of the quadriceps femoris 364 during single- and multi-joint exercises. European Journal of Applied Physiology 116 (5):1031-

365 1041. doi:10.1007/s00421-016-3363-5

366 Escamilla RF. 2001. Knee biomechanics of the dynamic squat exercise. Medicine and Science in

367 Sports and Exercise 33 (1):127-141. doi:10.1097/00005768-200101000-00020

368 Franchi, M. V., Longo, S., Mallinson, J., Quinlan, J. I., Taylor, T., Greenhaff, P. L., \& Narici, M.

369 V. (2018). Muscle thickness correlates to muscle cross-sectional area in the assessment of

370 strength training-induced hypertrophy. Scandinavian Journal of Medicine \& Science in Sports

371 28(3), 846-853. doi:10.1111/sms.12961

372 Häkkinen K, Kallinen M, Izquierdo M, Jokelainen K, Lassila H, Mälkiä E, Kraemer WJ, Newton

373 RU, Alen M. 1998. Changes in agonist-antagonist EMG, muscle CSA, and force during strength 
374 training in middle-aged and older people. Journal of Applied Physiology (Bethesda, Md : 1985)

37584 (4):1341-1349. doi:10.1152/jappl.1998.84.4.1341

376 Kritz M, Cronin J, Hume P. 2009. The bodyweight squat: A movement screen for the squat

377 pattern. Strength \& Conditioning Journal 31 (1):76-85

378 Kubo K, Ikebukuro T, Yata H. 2019. Effects of squat training with different depths on lower

379 limb muscle volumes. European Journal of Applied Physiology 119 (9):1933-1942

380 Mangine GT, Fukuda DH, LaMonica MB, Gonzalez AM, Wells AJ, Townsend JR, Jajtner AR,

381 Fragala MS, Stout JR, Hoffman JR. 2014a. Influence of gender and muscle architecture

382 asymmetry on jump and sprint performance. Journal of Sports Science \& Medicine 13 (4):904-

383911

384 Mangine GT, Hoffman JR, Gonzalez AM, Jajtner AR, Scanlon T, Rogowski JP, Wells AJ,

385 Fragala MS, Stout JR. 2014b. Bilateral differences in muscle architecture and increased rate of

386 injury in national basketball association players. Journal of Athletic Training 49 (6):794-799.

387 doi:10.4085/1062-6050-49.3.60

388 Marchetti PH, Jarbas da Silva J, Jon Schoenfeld B, Nardi PS, Pecoraro SL, D'Andréa Greve JM,

389 Hartigan E. 2016. Muscle Activation Differs between Three Different Knee Joint-Angle

390 Positions during a Maximal Isometric Back Squat Exercise. Journal of Sports Medicine

391 (Hindawi Publishing Corporation) 2016:3846123. doi:10.1155/2016/3846123

392 Methenitis SK, Zaras ND, Spengos KM, Stasinaki AN, Karampatsos GP, Georgiadis GV, Terzis

393 GD. 2016. Role of Muscle Morphology in Jumping, Sprinting, and Throwing Performance in

394 Participants With Different Power Training Duration Experience. Journal of Strength and

395 Conditioning Research 30 (3):807-817. doi:10.1519/jsc.0000000000001147

396 Nimphius S, McGuigan MR, Newton RU. 2012. Changes in muscle architecture and

397 performance during a competitive season in female softball players. The Journal of Strength \&

398 Conditioning Research 26 (10):2655-2666

399 Noorkoiv M, Nosaka K, Blazevich AJ. 2010. Assessment of quadriceps muscle cross-sectional

400 area by ultrasound extended-field-of-view imaging. Eur J Appl Physiol 109 (4):631-639.

401 doi:10.1007/s00421-010-1402-1

402 Robertson DG, Wilson JM, St Pierre TA. 2008. Lower extremity muscle functions during full

403 squats. Journal of Applied Biomechanics 24 (4):333-339. doi:10.1123/jab.24.4.333

404 Schoenfeld BJ. 2010. Squatting kinematics and kinetics and their application to exercise

405 performance. Journal of Strength and Conditioning Research 24 (12):3497-3506.

406 doi:10.1519/JSC.0b013e3181bac2d7

407 Secomb JL, Lundgren LE, Farley OR, Tran TT, Nimphius S, Sheppard JM. 2015. Relationships

408 between lower-body muscle structure and lower-body strength, power, and muscle-tendon

409 complex stiffness. The Journal of Strength \& Conditioning Research 29 (8):2221-2228

410 Seitz LB, Trajano GS, Haff GG, Dumke CC, Tufano JJ, Blazevich AJ. 2016. Relationships

411 between maximal strength, muscle size, and myosin heavy chain isoform composition and

412 postactivation potentiation. Applied Physiology, Nutrition, and Metabolism = Physiologie

413 appliquee, nutrition et metabolisme 41 (5):491-497. doi:10.1139/apnm-2015-0403 
414 Seymour, J. M., Ward, K., Sidhu, P. S., Puthucheary, Z., Steier, J., Jolley, C. J., ... \& Moxham, J. 415 (2009). Ultrasound measurement of rectus femoris cross-sectional area and the relationship with 416 quadriceps strength in COPD. Thorax 64(5), 418-423. doi:10.1136/thx.2008.103986

417 Suchomel TJ, Stone MH. 2017. The relationships between hip and knee extensor cross-sectional 418 area, strength, power, and potentiation characteristics. Sports 5 (3):66

419 Ten Haaf, D., Hemmen, B., van de Meent, H., \& Bovend'Eerdt, T. J. (2017). The magnitude and

420

421

422

423

424

425

426

427

428

429

430

431

432

433

434

435

436

437

438

439

440

441

442

443

444 time course of muscle cross-section decrease in intensive care unit patients. American Journal of Physical Medicine \& Rehabilitation 96(9), 634-638. doi: 10.1097/PHM.0000000000000711 Toumi H, Best T, Pinti A, Lavet C, Benhamou C, Lespessailles E. 2013. The role of muscle strength \& activation patterns in patellofemoral pain. Clinical Biomechanics 28 (5):544-548 Toutoungi DE, Lu TW, Leardini A, Catani F, O'Connor JJ. 2000. Cruciate ligament forces in the human knee during rehabilitation exercises. Clinical Biomechanics (Bristol, Avon) 15 (3):176187. doi:10.1016/s0268-0033(99)00063-7

Vigotsky AD, Bryanton MA, Nuckols G, Beardsley C, Contreras B, Evans J, Schoenfeld BJ. 2019. Biomechanical, Anthropometric, and Psychological Determinants of Barbell Back Squat Strength. Journal of Strength and Conditioning Research 33 Suppl 1:S26-s35. doi:10.1519/jsc.0000000000002535

Watanabe K, Akima H. 2011. Validity of surface electromyography for vastus intermedius muscle assessed by needle electromyography. Journal of Neuroscience Methods 198 (2):332335. doi:10.1016/j.jneumeth.2011.03.014

Wilhelm EN, Rech A, Minozzo F, Radaelli R, Botton CE, Pinto RS. 2014. Relationship between quadriceps femoris echo intensity, muscle power, and functional capacity of older men. Age (Dordrecht, Netherlands) 36 (3):9625. doi:10.1007/s11357-014-9625-4

Zebis MK, Andersen LL, Bencke J, Kjaer M, Aagaard P. 2009. Identification of athletes at future risk of anterior cruciate ligament ruptures by neuromuscular screening. The American Journal of Sports Medicine 37 (10):1967-1973. doi:10.1177/0363546509335000

van Melick, N., Meddeler, B. M., Hoogeboom, T. J., Nijhuis-van der Sanden, M. W., \& van Cingel, R. E. (2017). How to determine leg dominance: The agreement between self-reported and observed performance in healthy adults. PloS one 12 (12), e0189876. doi:

10.1371/journal.pone.0189876 


\section{Table 1 (on next page)}

Sample characteristics.

BH- body height; BM- body mass; BMI- body mass index; SMM- skeletal muscle mass; PBF percent body fat; LSMM- dominant leg skeletal muscle mass 
1 Table 1:

2 Sample characteristics.

\begin{tabular}{ll}
\hline Variables & Mean \pm SD \\
\hline Age (years) & $24.1 \pm 1.7$ \\
BH $(\mathrm{m})$ & $1.75 \pm 0.08$ \\
BM $(\mathrm{kg})$ & $70.4 \pm 12.03$ \\
BMI $\left(\mathrm{kg} / \mathrm{m}^{2}\right)$ & $22.7 \pm 2.3$ \\
SMM $(\mathrm{kg})$ & $33.23 \pm 7.60$ \\
PBF $(\%)$ & $16.48 \pm 7.30$ \\
LSMM $(\mathrm{kg})$ & $9.3 \pm 1.32$
\end{tabular}

3 BH- body height; BM- body mass; BMI- body mass index; SMM- skeletal muscle mass; PBF

4 percent body fat; LSMM- dominant leg skeletal muscle mass

5 


\section{Table 2 (on next page)}

Cross-sectional area of quadriceps muscles and leg muscle mass between males and females.

*- indicates a significant gender difference $(p<0.05)$; **- indicates a significant gender difference $(p<0.01)$ 
1 Table 2:

2 Cross-section area of quadriceps muscles and leg muscle mass between males and females.

\begin{tabular}{lcc}
\hline & Males & Females \\
\cline { 2 - 3 } Muscles & Mean \pm SD & Mean \pm SD \\
\hline Rectus Femoris $\left(\mathrm{mm}^{2}\right)$ & $383.67 \pm 79.73^{*}$ & $285.71 \pm 95.49$ \\
Vastus Intermedius $\left(\mathrm{mm}^{2}\right)$ & $333.44 \pm 83.20^{* *}$ & $205,71 \pm 46.59$ \\
Vastus Medialis $\left(\mathrm{mm}^{2}\right)$ & $337.11 \pm 67.57 * *$ & $242,29 \pm 39.69$ \\
Vastus Lateralis $\left(\mathrm{mm}^{2}\right)$ & $374.67 \pm 72.45$ & $321.00 \pm 40.59$ \\
Quadriceps Femoris $\left(\mathrm{mm}^{2}\right)$ & $1428.89 \pm 223.04 * *$ & $1054.71 \pm 204.48$ \\
LSMM (kg) & $10.68 \pm 1.02 * *$ & $7.52 \pm 1.15$ \\
\hline
\end{tabular}

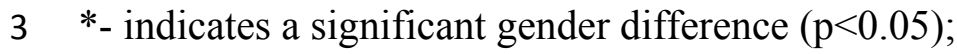

$4 * *$ - indicates a significant gender difference $(\mathrm{p}<0.01)$ 
Table 3(on next page)

Correlation between external load and cross- section area of Quadriceps Femoris muscles. 
1 Table 3:

2 Correlation between external load and cross- section area of Quadriceps Femoris muscles.

\begin{tabular}{lcccc}
\hline & \multicolumn{2}{c}{ Parallel Squat } & \multicolumn{2}{c}{ Deep Squat } \\
\cline { 2 - 5 } Muscles & $r$ & $\mathrm{p}$ & $r$ & $\mathrm{p}$ \\
\hline Rectus Femoris & .408 & .117 & .315 & .235 \\
Vastus Intermedius & $\mathbf{. 5 4 6}$ &. $\mathbf{0 2 9}$ & $\mathbf{. 5 0 9}$ & $\mathbf{. 0 4 4}$ \\
Vastus Medialis & $\mathbf{. 7 5 4}$ & $\mathbf{. 0 0 1}$ & $\mathbf{. 7 0 6}$ & $\mathbf{. 0 0 2}$ \\
Vastus Lateralis & $\mathbf{. 6 6 9}$ & $\mathbf{. 0 0 5}$ & $\mathbf{. 5 9 8}$ & $\mathbf{. 0 1 4}$ \\
Quadriceps Femoris & $\mathbf{. 6 7 2}$ & $\mathbf{. 0 0 4}$ & $\mathbf{. 5 9 9}$ & $\mathbf{. 0 1 4}$ \\
\hline
\end{tabular}

3

4 
Figure 1

Study protocol

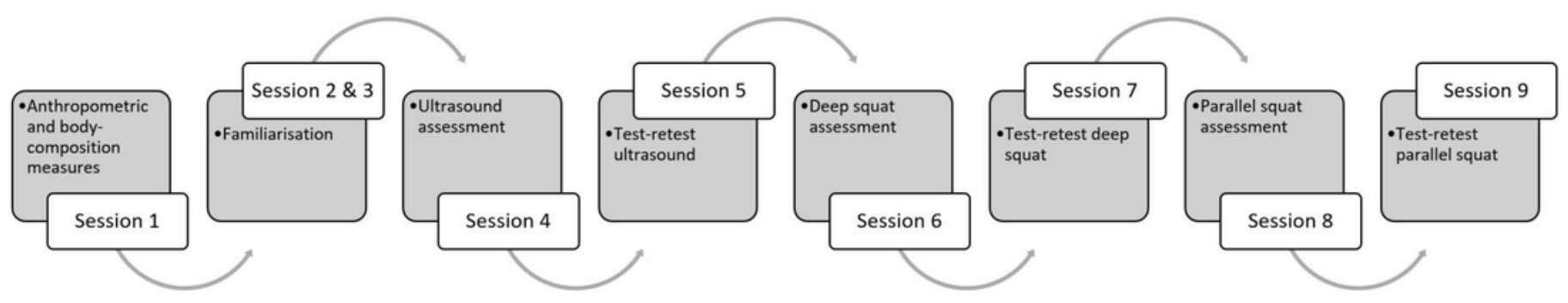


Figure 2

Illustration of participants performing the deep squat and parralel squat.
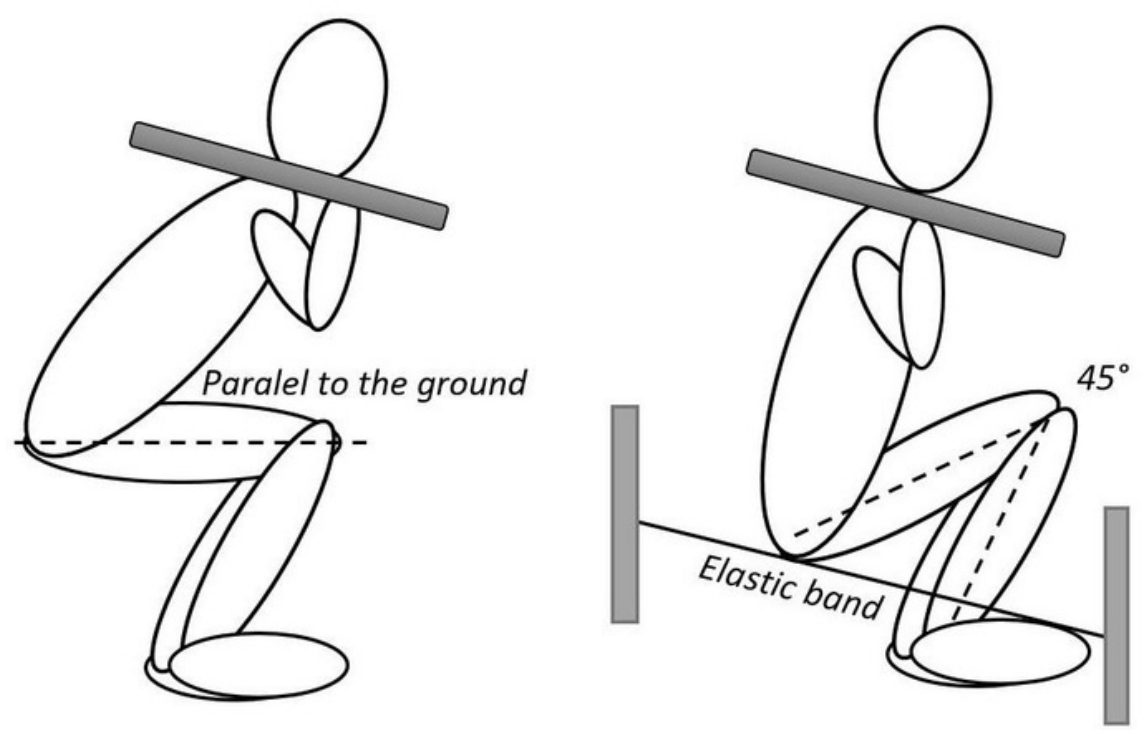
Figure 3

Deep squat and parallel squat external load values for males and females.

**- indicates a significant squat variation difference $(p<0.01)$; \#- indicates a significant gender difference $(p<0.01)$.

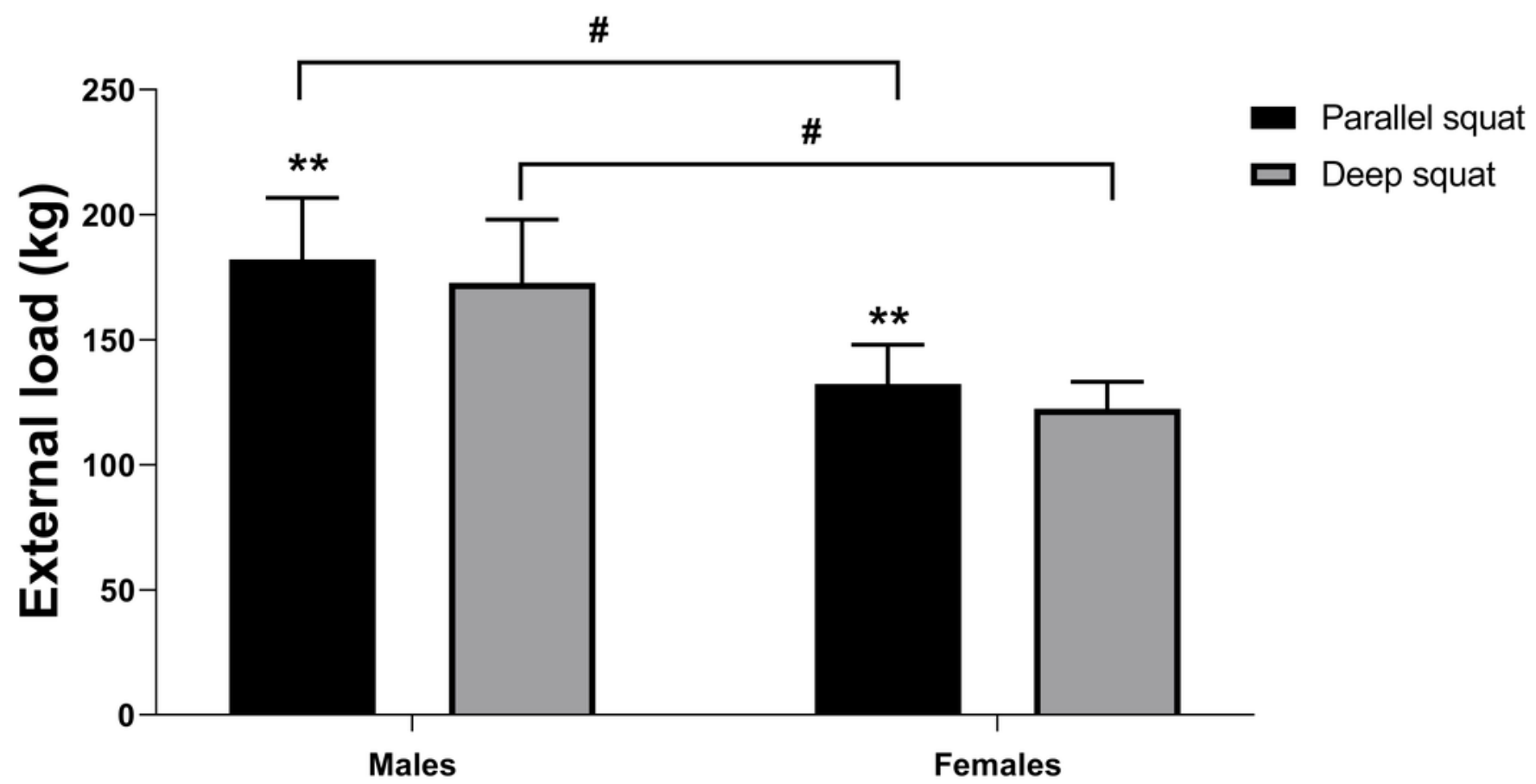


Figure 4

External load prediction for parallel (panel A) and deep squat (panel B) based on the cross-section area of vastus medialis.

VM - vastus medialis. 


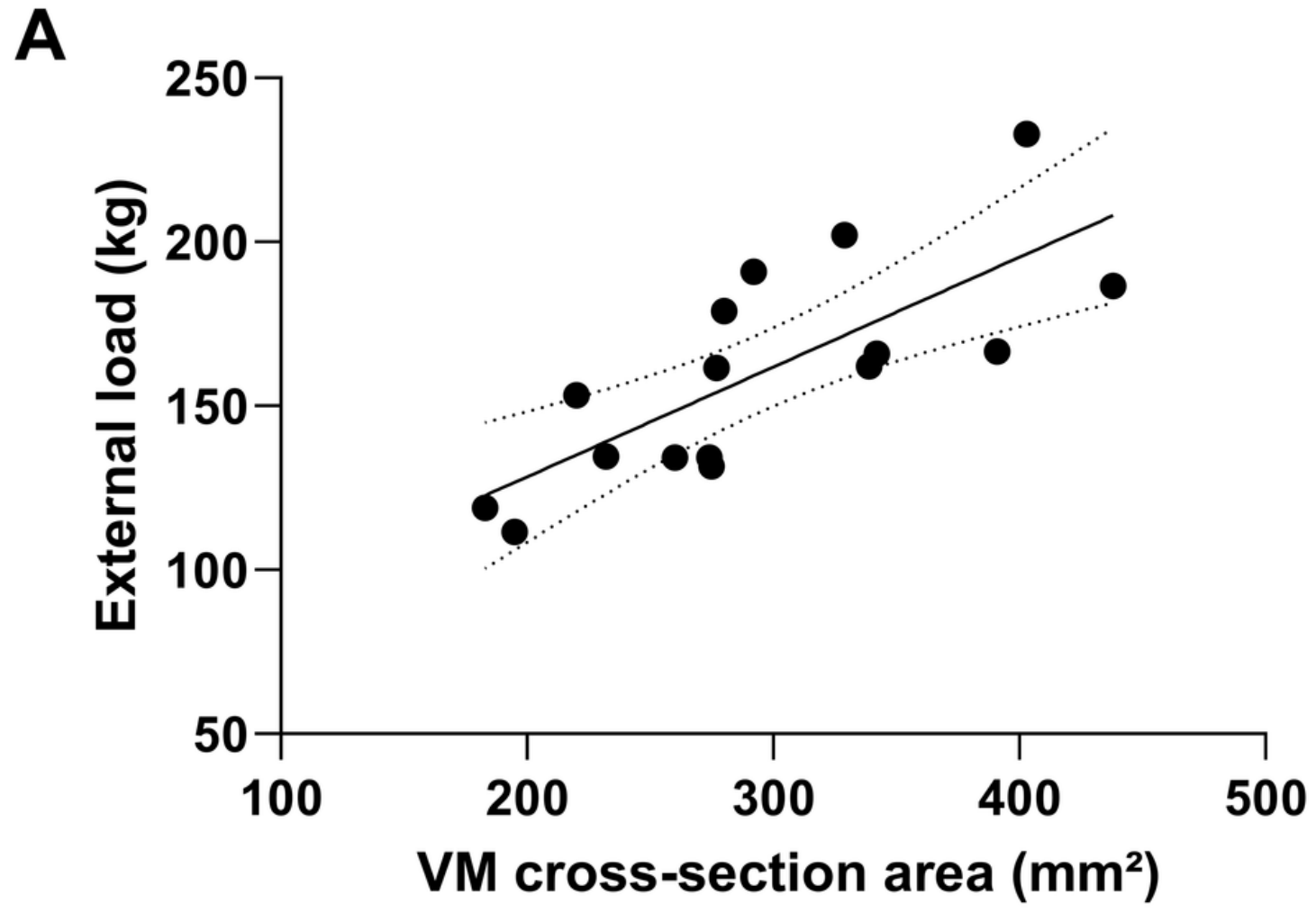

B

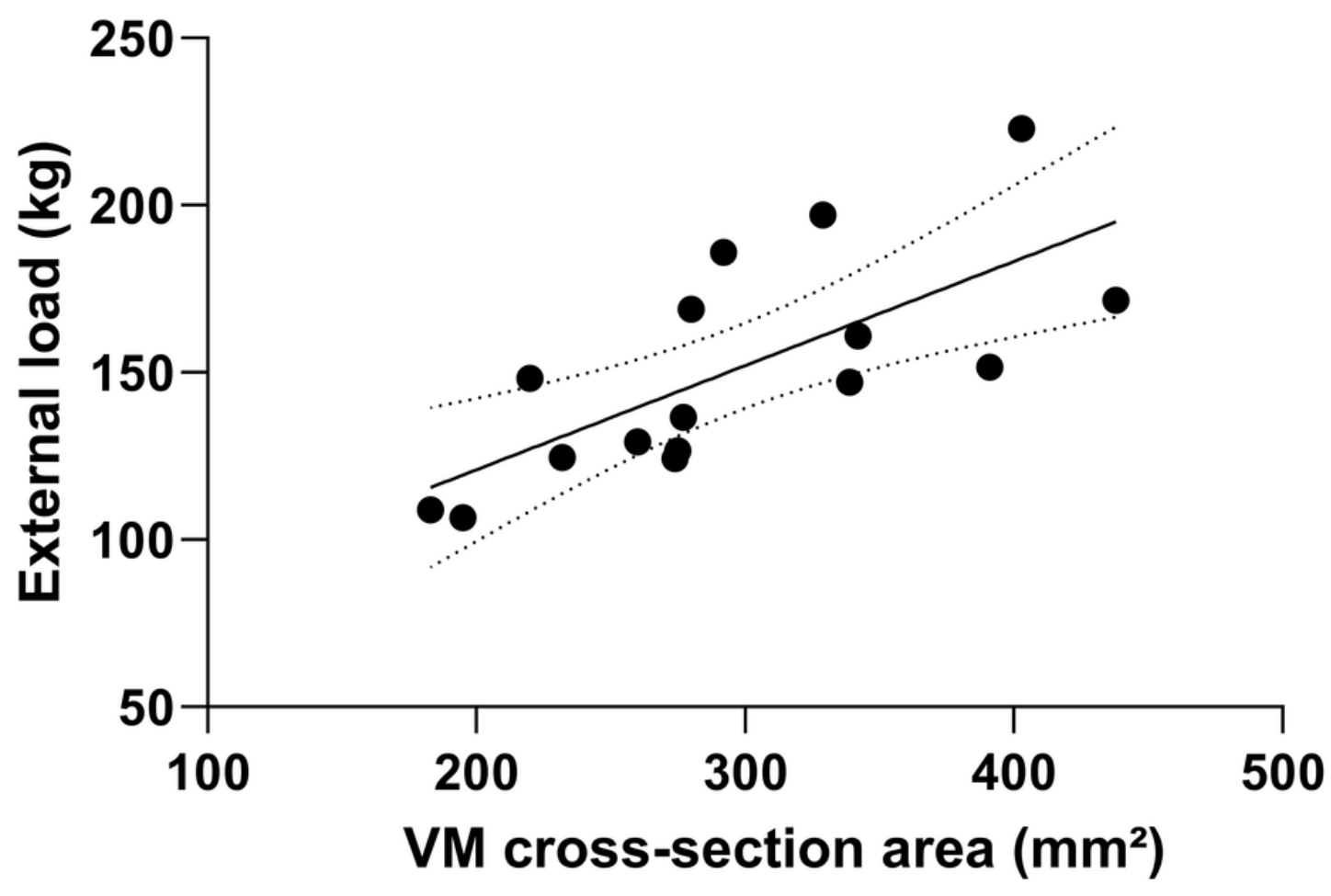

